OPTICAL-PHONON-MEDIATED CHARGE TRANSPORT IN SUBSTITUTED MORPHOLINIUM TCNQ 2 SALTS

\author{
G.J. KRAMER, H.B. BROM AND L.J. DE JONGH
}

Kamerlingh Onnes Laboratorium, Rijksuniversiteit te Leiden, Postbus 9506, 2300 RA Le1den The Netherlands

Substituted morpholinium $\mathrm{TCNQ}_{2}$ compounds exhib1t some universal features with respect to the field, frequency and temperature dependence of the conductivity. These characteristics are different from the closely related morpholinfum $\mathrm{TCNQ}_{1}$ compounds. We show that a natural way to account for these phenomena comes about by careful examination of the order parameter space, $i$.e. the space created by the two different order parameters which together determine the gap at the Fermi surface: (1) the regular Pelerls dimerization of the acceptor-acceptor overlap and (11) a coherent shift of the donor molecules with respect to the acceptors. By describing small revolutions through parameter space (realized by phase coupling of two different phonons producing the above mentioned gap) the ground state electron gas is shifted with respect to the lattice, thereby producing a current. The model allows for a qualitative description of conductivityrelated phenomena in these systems.

\section{INTRODUCTION AND EXPERIMENTAL}

The class of TCNQ compounds with subst1tuted morpholinium as donor, has been extensively studied over the past decade, expecial1y MEM(TCNQ) 2 which exhibits both a metal to semiconductor transition and a Spin-Peierls transition $^{1}$. In spite of this long-standing interest, the conductivity mechanism is still poorly understood. Below we present and discuss experiments on the dependence of the conductivity on both electric field strength and frequency. The latter type of experiments have proven to be useful in discriminating between conduction mechanisms in other 1-d compounds 1ike $\mathrm{NbSe}_{3}$ and $\mathrm{Qn}-\mathrm{TCNQ}_{2}{ }^{2}$.

Figure 1 shows a typical example of the field and frequency dependence of a $\mathrm{M}-\mathrm{TCNQ}_{2}$ compound. Both experimental curves can be fitted to the same empirical formula

$$
\sigma(x)=\sigma_{0}\left(1+A \cdot \exp \left[-x_{m} / x^{\alpha}\right]\right)
$$

where $x$ can be elther electric field or frequency. For all compounds the temperature dependence of the conductivity is activated, with activation energies of the order of

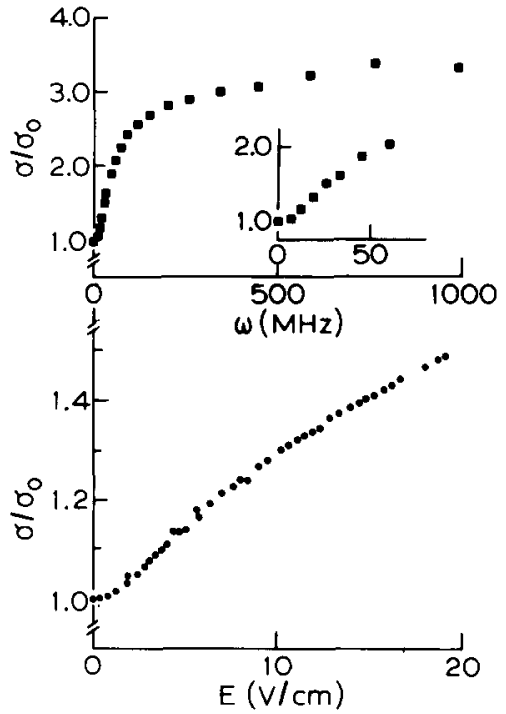

Fig. 1 Frequency and field dependence of the conductivity of methy1-ethyl-thlomorpholinium at room temperature. The insert shows the lowfrequency data from which the similarity with the fleld-dependence is apparent. 
$0.1 \mathrm{eV}$. As regards the fleld-dependence we find that eq.(1) remains valid with temperature Independent values for $A, \alpha$ and $x_{m}$, indicative of a single conductivity mechanism. The functional form of (1) shows - for $\alpha=1$ that the conductivity is in part activated in field and frequency. For all compounds which exhibit the above mentioned behaviour, the values for $\alpha$ were always markedly smaller than $1^{3}$. Th1s can be explained by assuming a distribution in $x_{m}$. Indeed, $1 t$ was found ${ }^{4}$ that the value of $\alpha$ can be related to the amount of disorder in the donor system. However, even for systems with a perfectly regular donorand TCNQ-lattice, values as low as 0.5 were found for $\alpha$. Another important observation is the absence of any such effects in compounds with a $1: 1$ donor to acceptor ratio.

Below we shall discuss a model for charge transport, which employs the unique features of charge transfer salts with $1: 2$ donor to acceptor ratio, and which provides for a qualitative understanding of our observations.

\section{OPTICAL-PHONON-MEDIATED CHARGE TRANSPORT}

In a charge transfer salt with $1: 2$ donor to acceptor ratio; the electron density at the Fermi-surface may be reduced by the deformation of the lattice with a periodicity of two TCNQ-spacings ${ }^{5}$. This can be done in two fundamentally different ways. Firstly, the TCNQspacing may alternate, which is the usual Peierls mechanism. Secondly, the donor molecules may undergo a $k=0$ shift with respect to the TCNQ-neighbours, resulting in a alternation of the energy of the TCNQ valence orbital, which gives rise to a gap at the Fermilevel and a modulated electron density with a periodicity of two TCNQ-spacings. The hamiltonian for the system reads:

$$
\pi=\sum_{1}\left[t+(-1)^{1} \tau\right]\left(C_{1}^{+} C_{1+1}+C_{1+1}^{+} C_{1}\right)+\underset{1}{\Sigma(-1)^{1} E} C_{1}^{+} C_{1}
$$

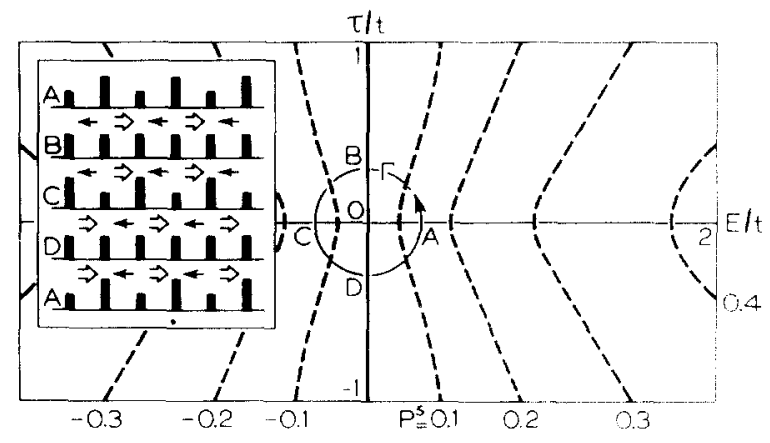

Fig. 2 Order parameter space for $\mathrm{M}^{-} \mathrm{TCNQ}_{2}$ compounds. The dotted 11 nes are contours of equal staggered electron density. The insert shows the changes in the ground state electron configuration resulting from proceeding along $\Gamma$. Eat (thin) arrows indicate large (small) overlaps.

where $\tau$ and $E$ represent the mentioned deformations, which together span up a 2-dimensional order parameter space. Figure 2 shows this parameter space with the staggered electron occupation for the ground state, defined as

$$
\rho^{s}=\frac{1}{2}+\rho_{2 n}=\frac{1}{2}-\rho_{2 n+1}
$$

Fig. 2 is a good starting point for the formulation of the proposed charge transport mechanism. Let us assume the ground state electron gas to be governed by $E$ and $\tau$, and consider what happens upon passing along the trajectory $A B C D A$ in $\mathrm{fig} .2$. The original staggered electron occupation diminishes at first along $A B$ and then builds up again to a negative value over $B C$. All the way along $A B C$ a net charge transport occurs from even- to the neighbouring odd-numbered sites. This transport has a left-right asymetry since all the way along $A B C, \tau>0$. Because the transition probability for an electron hop is proportional to the square of the overlap, this results in a net rightward charge transport, amounting to

$$
\delta Q=\delta \rho^{S}(P(*)-P(\rightarrow))=\delta \rho^{s} \cdot 2 \tau / t
$$


As the reader may verify, the same rightward transport occurs in the second half of the loop (CDA) and hence

$$
\delta Q=2 \underset{\Gamma}{2} \mathrm{~d} \rho^{8} \frac{\tau}{\mathrm{t}} \neq 0
$$

This implies that cooperative charge transport results from coherent variation of the two orderparameters $E$ and $\tau$. This in turn assures $a$ coupling between the $\mathrm{E}$ - and $\tau$-mode $1 \mathrm{~s}$ coupled to the time dependence of the phase of the $\mathrm{E}-$ mode in such a way that charge is transported in the direction favoured by the electric field.

At this point we want to draw attention to the relation between the above proposed model and CDW transport in 1ncommensurate 1-d metals. In the latter system, the slipping of the phase of the CDW order parameter allows for cooperative charge transport ${ }^{2}$. In the present system a similar phase slip of elther one of the order parameters alone would not contribute to the conductivity because of the high order of commensurability. As argued above, a jolnt, coherent modulation of both order parameters removes the objection of commensurability pinning by opening a new part of phase space. Another important difference is that in the commensurate system with two different order parameters, the total energy of the condensate is not - as for the regular CDW system - Independent of the place on the circle in fig. 1. An Inevitable consequence of our model is that in the $\mathrm{M}-\mathrm{TCNQ}_{2}$ system $^{3}$, the contribution of cooperative charge transport is always temperature activated, in contrast to the idea of Fröhlich collective modes underlying the regular CDW system, which would give a contribution even at zero temperature.

From this the conclusion can be drawn that optical phonons should be responsible for the conductivity. Small, local fluctuations in the global value of the order parameters $E$ and $\tau$ are brought about by optical phonons. These fluctuations cause tiny revolutions in parameter space, which leads to cooperative charge transport. Consequently, the two different phonon modes get phase-coupled in the presence of an electric field ${ }^{6}$. This model does make some predictions for the electrical conductiv1ty, which are indeed observed. Firstly, one finds a temperature activated conductivity. In the present treatment of the problem the activation energy is related to the optical phonon energles, which seems reasonable. Secondly, preliminary calculations for the fleld dependence of the conductivity yleld both saturation at high flelds and scaling of the field dependence with $\sigma_{0}$, in accordance with the measurements ${ }^{4}$.

In conclusion: the model of optical-phononmediated charge transport, which specifically employs the unique structure of $1: 2$ donor-acceptor systems has been shown to account for some conductivity-related phenomena which can not be accounted for by "standard" models for 1-D conductors.

\section{REFERENCES}

1. S. Huizlnga, J. Kommandeur, G.A. Sawatzky, K. Kopinga, W.M.J. de Jonge, Proc. Int. Conf. on Q1D Cond., Dubrovnik, 1978, Vol. 96 of Lecture Notes in Physics (Springer, Ber11n, 1978), p.45.

2. G. Grüner and A. Zett1, Phys. Rep. 119, 117 (1985).

3. G.J. Kramer, J.L. Joppe, H.B. Brom, L.J. de Jongh and J.L. de Boer in the proceedings of the ICSM'86, to be published in Synth. Metals.

4. G.J. Kramer, H.B. Brom, L.J. de Jongh and J.L. de Boer, Festkörperprobleme, Advances in Solid State Physics XXV, 167 (1985).

5. We assume that the on-site electronelectron repulsion is much larger than the bandwidth, which is the case for $\mathrm{M}-\mathrm{TCNQ}_{2}$ compounds.

6. A detalled treatment can be found in G.J. Kramer, H.B. Brom and L.J. de Jongh, proceeding 8 of the ICSM' 86 , to be published in Synth. Metals. 